\title{
Study of optimal exposure windows using 320-Detector rows dynamic volume CT
}

\author{
This article was published in the following Dove Press journal: \\ Reports in Medical Imaging \\ 3 December 2010 \\ Number of times this article has been viewed
}

\author{
Gang Sun' \\ Min $\mathrm{Li}^{\prime}$ \\ $\mathrm{Li} \mathrm{Li'}$ \\ Guo-ying $\mathrm{Li}^{\prime}$ \\ Zhi-wei Jing ${ }^{2}$ \\ 'Departments of Medical Imaging, \\ ${ }^{2}$ Medical Statistics, Jinan Military \\ General Hospital, Shandong Province, \\ China
}

Correspondence: Gang Sun or Min Li Department of Medical Imaging, Jinan Military General Hospital, No 25 Shifan Road, Jinan, Shandong Province, China 25003।

Tel +8653I 5I 666277

Fax +8653151666486

Email cjr.sungang@vip.163.com;

limin22000@yahoo.com.cn
Abstract: The purpose of this study was to determine the optimal electrocardiographic (ECG) pulsing windows and evaluate the effect on reduced dose and accuracy using 320-detector rows dynamic volume computed tomography (DVCT). A total of 170 patients were prospectively studied. The optimal reconstruction windows were analyzed in 76 patients scanned using retrospective ECG gating. Forty-seven patients were scanned by the predicted triggering windows. The optimal positions of exposure intervals according to different heart rates were evaluated. Optimal image quality, radiation dose, and diagnostic accuracy were then investigated by applying optimal triggering windows. The optimal ECG pulsing windows were determined as follows: when heart rate was $<70$ beats per minute, the exposure windows should be preset at $60 \%-80 \%$; for a heart rate $70-90$ beats per minute at $70 \%-90 \%$; and for a heart rate $\geq 90$ beats per minute at $30 \%-50 \%$. The radiation dose for patients scanned with prospective ECG gating was significantly lower (5.9 versus $12.9 \mathrm{mSv}, P<0.001$ ). However, because two or three heart beats were needed when heart rate was $>70$ beats per minute, the radiation dose increased with increasing heart rate for both retrospective and prospective ECG gating $(\mathrm{r}=0.64, P<0.001$ and $\mathrm{r}=0.59, P<0.001$, respectively). On the basis of a per segment analysis, overall sensitivity was $98.0 \%$ (49/50), specificity was $99.2 \%(602 / 607)$, the positive predictive value was $90.7 \%$ (49/54), and the negative predictive value was $99.8 \%$ (602/603). In conclusion, DVCT has the potential to provide high image quality across a wide range of heart rates using an optimized ECG pulsing window. However, it is recommended to control heart rate below 70 beats per minute, if possible, to decrease the radiation dose.

Keywords: dynamic volume computed tomography, coronary angiography, optimal exposure window, heart rate, radiation dose

\section{Introduction}

In less than a decade, cardiac computed tomography (CT) technology has progressed to multislice CT and dual-source CT with wide cardiac coverage and increased temporal resolution, which has resolved problems caused by high heart rates. However, since Einstein et al estimated a significant number of potential radiation-induced neoplasms arising from coronary CT angiography, ${ }^{1}$ the high effective dose and potential adverse consequences of coronary CT angiography have aroused more attention and limited the general applicability of this test. ${ }^{2-5}$ To reduce the patient dose, electrocardiographic (ECG) gating in coronary CT angiography has advanced from being a retrospective procedure, which needs exposure during the whole R-R interval, to prospective ECG gating. However, prospective ECG gating is limited by the fact that CT data are acquired 
over only a fraction of the R-R interval, and requires careful definition of the optimal ECG pulsing window. ${ }^{6-12}$

Since the early application of multislice CT in the field of noninvasive coronary angiography, CT has been improved from four detectors to $16,64,128$, and 256 detectors. The diagnostic accuracy has also been improved greatly with the increasing width of detector array coverage. However, the coverage is still too narrow to cover the whole heart in one heart beat. The recent introduction of 320-detector rows dynamic volume CT (DVCT) enables whole heart coverage within one heart beat (no table movement). Using prospective ECG gating, DVCT can significantly reduce the radiation dose and the amount of contrast agent needed. ${ }^{13-18}$ However, the study by Dewey and colleagues only focused on patients with a low heart rate. For higher heart rates, exposure windows were generally expanded to cover both the systolic and diastolic duration to preserve optimal intervals, but this comes at the cost of a higher radiation dose. ${ }^{17} \beta$-blockers are generally administered to lower heart rate for better image quality and lower the effective dose, but this is ineffective in about $20 \%$ of patients, and may have adverse effects. ${ }^{19,20}$ Thus, it is critical to evaluate the optimal exposure windows when heart rate cannot be controlled or contraindications exist. For DVCT, when heart rate exceeds 80 beats per minute, a three heart beat acquisition scan mode is used, which can improve the temporal resolution to $58.3 \mathrm{msec}$. Our hypothesis was that the optimal reconstruction intervals at a higher heart rate were different from previous CT. Thus, the purpose of our study was to evaluate prospectively the heart rate dependency of image quality by incorporating the analysis of regulation of the optimal reconstruction windows and estimate the reduced effective doses and diagnostic accuracy by applying the predicted triggering windows.

\section{Materials and methods Study patients}

Our study protocol was approved by the local Ethics Committee. Informed consent, which included information about the risk of radiation and iodine allergy, were obtained from all patients.

For evaluation of the optimal exposure intervals, 119 patients whose coronary vessels and cardiac function needed assessment were prospectively enrolled between December 2008 and October 2009. Exclusion criteria were as follows: allergy to iodinated contrast agents, renal insufficiency (creatinine level $>120 \mu \mathrm{mol} / \mathrm{L}$ ), pregnancy and hemodynamic instability, abnormal origin and course of the coronary vessels, and presence of arrhythmias. ${ }^{21-23}$
Patients who underwent stent-grafting and bypass surgery were excluded from the study. After exclusion of 41 patients and two further patients who could not be enrolled because of refusal or withdrawal of consent, the final study group included 76 patients (31 women and 45 men, mean age, $65.3 \pm 10.2$ years, age range $39-91$ years). Oral $\beta$-blockade ( $50 \mathrm{mg}$ atenolol) was given to patients with a heart rate $>70$ beats per minute (except for seven patients with a contraindication, including patients with atrioventricular block, bronchial asthma, and obstructive emphysema). All patients with heart rate $>70$ beats per minute after administration of $\beta$-blockade and patients with contraindications were included in our study.

An ECG tube current modulation was used to achieve dose reduction. The full tube current was set at 30\%-90\% of the R-R interval. Outside the ECG gating window, the tube current was reduced to $30 \%$ of the full current. From December 2009 to April 2010, 51 patients who were scheduled for clinically indicated invasive coronary angiography were scanned by applying the predicted triggering windows. After exclusion of the patients mentioned earlier, 47 patients were included. Patient characteristics are given in Table 1.

Table I Demographic data

\begin{tabular}{|c|c|c|}
\hline Characteristic & Retrospective & Prospective \\
\hline Enrolled patients, $n$ & 119 & 51 \\
\hline \multicolumn{3}{|l|}{ Clinical feature } \\
\hline Typical angina, $\mathrm{n}$ & 42 & 37 \\
\hline Atypical angina, $n$ & 31 & 10 \\
\hline Abnormal & 20 & I \\
\hline \multicolumn{3}{|l|}{ electrocardiogram, $n$} \\
\hline Myocardial infarction, $\mathrm{n}$ & 26 & 3 \\
\hline Excluded patients, $\mathrm{n}$ & 43 & 4 \\
\hline $\begin{array}{l}\text { Allergy to iodinated } \\
\text { contrast agents, } n\end{array}$ & 2 & \\
\hline Renal insufficiency, $\mathrm{n}$ & I & \\
\hline Pregnancy, $\mathrm{n}$ & I & \\
\hline Hemodynamic instability, $n$ & I & \\
\hline $\begin{array}{l}\text { Abnormal origin } \\
\text { and course, } n\end{array}$ & 7 & \\
\hline $\begin{array}{l}\text { Stent-grafting and bypass } \\
\text { surgery, } \mathrm{n}\end{array}$ & 4 & I \\
\hline Heart rate variability & 25 & 3 \\
\hline$>20$ bpm, $\mathrm{n}$ & & \\
\hline $\begin{array}{l}\text { Denied written informed } \\
\text { consent, } \mathrm{n}\end{array}$ & 2 & \\
\hline Final patients, $\mathrm{n}$ & 76 & 47 \\
\hline Age (years) & $65.3 \pm 10.2$ & $65.5 \pm 10.7$ \\
\hline Male/female ratio & $45 / 31$ & $36 / 11$ \\
\hline Body mass index $\left(\mathrm{kg} / \mathrm{m}^{2}\right)$ & $23.8 \pm 3.6$ & $23.5 \pm 3.9$ \\
\hline $\begin{array}{l}\text { Average heart rate during } \\
\text { scan }(\mathrm{bpm})\end{array}$ & $71.2 \pm 13.2$ & $74.8 \pm 11.8$ \\
\hline
\end{tabular}

Abbreviation: bpm, beats per minute. 


\section{CT protocol}

All CT examinations were performed using DVCT (Aquilion One, Toshiba, Nasu, Japan) with a gantry rotation speed of $350 \mathrm{msec}$ and detector collimation of $320 \times 0.5 \mathrm{~mm}$. Nitroglycerin $0.4 \mathrm{mg}$ was administered two minutes before scanning in the absence of contraindications (eg, hypotension, current use of nitrate medications, migraine sensitive to nitrates). The scanning range was set from the level of the tracheal bifurcation to the diaphragm to cover the entire heart.

A 40-60 mL bolus of iohexol (Omnipaque 350, Amersham Health [now GE Healthcare], Shanghai, China) was injected into an antecubital vein through an 18-gauge catheter at an injection rate of $4-6 \mathrm{~mL} / \mathrm{sec}$ followed by $50 \mathrm{~mL}$ saline solution continuously. The enhanced scan was controlled by means of bolus tracking (SUREStart, Toshiba), and the trigger threshold was set at $180 \mathrm{HU}$ in the descending aorta at the same level as in the aorta root. Scanning parameters were a tube potential of $100-120 \mathrm{kV}$ and a tube current of $350-500 \mathrm{~mA}$. For body mass index (BMI) $<18,100 \mathrm{kV}$ and $350 \mathrm{~mA}$ were applied; for BMI 18-24, $100 \mathrm{kV}$ and $400 \mathrm{~mA}$; and for $\mathrm{BMI} \geq 24,120 \mathrm{kV}$ and $450-500 \mathrm{~mA}$.

If heart rate was $<70$ beats per minute at time of breathhold practice, image acquisition was manually adjusted using a one heart beat scan acquisition mode. For a heart rate of $70-80$ beats per minute, a two heart beat acquisition mode was used. For heart rate $\geq 80$ beats per minute, a three heart beat acquisition mode was necessary to increase temporal resolution. Using a multiheart beat acquisition mode, the temporal resolution could reach $87.5-58.3 \mathrm{msec}$. The ECG was digitally recorded during data acquisition and was stored for further data processing. All images were transferred to a separate workstation (Vitrea II FX, Vital Images, Minnetonka, MN).

\section{CT image reconstruction}

Image reconstruction was performed with a section thickness of $0.5 \mathrm{~mm}$ and an increment of $0.25 \mathrm{~mm}$. The reconstructed field of view was adjusted to encompass the heart exactly $(180 \times 180 \mathrm{~mm}$ to $240 \times 240 \mathrm{~mm})$. For evaluation of the optimal reconstruction intervals, image reconstruction based on relative timing was performed in $10 \%$ steps of the R-R interval in patients scanned with an ECG tube current modulation protocol. In those patients for whom a motion-free phase was not identified, image reconstruction was preceded by $1 \%$ intervals around the $10 \%$ intervals with fewest motion artifacts to determine the optimal image quality. Curve planar reconstruction was applied on the workstation to assess image quality of the coronary arteries.

\section{CT data analysis}

All CT data were interactively assessed by two readers (with 6-7 years' experience in cardiovascular radiology). Decisions were reached using consensus reading.

For analysis, we rated image quality on a per vessel basis, ie, for the right coronary artery, left anterior descending artery, and left circumflex artery. The score of segment with worst image quality represented the entire artery. ${ }^{24}$ This type of protocol was mainly focused on the success of the whole vessel, which was more feasible in clinical application. The reviewers assessed image quality semiquantitatively using a previously described 4-point ranking scale, ie, 1, excellent (no motion artifacts and clear delineation of the vessel); 2 , good (minor artifacts and mild blurring of the vessel); 3 , adequate, (moderate artifacts and moderate blurring without structure discontinuity); 4 , poor image quality (doubling or discontinuity in the course of the vessel preventing evaluation or vessel structures, not differentiable severe motion artifacts) as shown in Figure 1.25

The ECG protocol was analyzed by a third reviewer (LM) who was not involved in image quality assessment. Heart rate was defined as mean heart rate during examination.

\section{Evaluation of radiation dose for CT coronary angiography}

The dose length product (DLP) displayed on the dose report on the CT scanner was recorded. An effective dose (E) was obtained using the equation:

$\mathrm{E}=\mathrm{k} \times \mathrm{DLP}\left(\mathrm{k}=0.029 \mathrm{mSv} \times \mathrm{mGy}^{-1} \times \mathrm{cm}^{-1}\right.$, which was calculated specifically for DVCT) ${ }^{26}$

\section{Invasive coronary angiography}

Cardiac angiograms were performed using the conventional Judkins technique. ${ }^{27}$ Four views of the left coronary artery and two views of the right coronary artery were analyzed in consensus by two cardiologists, who were blinded to the CT results during analysis. Quantitative assessment of stenosis severity on angiograms was performed using the same criteria as those used for the CT data.

\section{Statistical analysis}

Statistical analysis was performed using statistical software (SPSS, version 16 for Windows; SPSS, Chicago, IL). A $P$ value of less than 0.05 indicates a statistically significant difference.

Quantitative variables were expressed as mean \pm standard deviation (SD) and categorical variables as frequencies or percentages. The comparison of image quality was tested by the Wilcoxon signed-rank test. Interobserver agreement for 

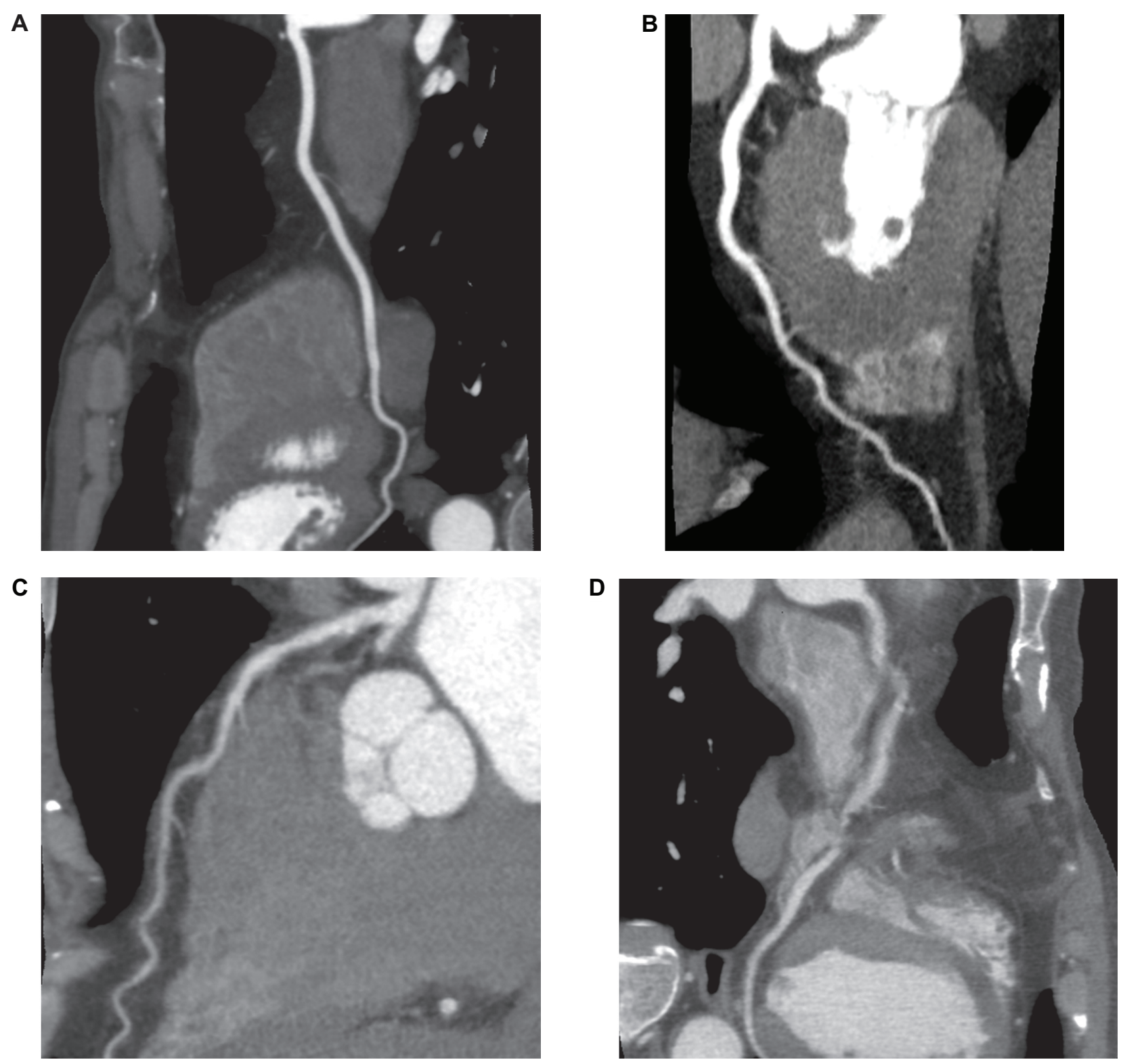

Figure I Curved multiplanar reformations of coronary vessels showed image quality score. A) Excellent image quality without motion artifacts; B) good image quality with minor artifacts and mild blurring (score 2); C) adequate image quality with moderate blurring (score 3); D) poor image quality with doubling or discontinuity.

the determination of image quality readout was calculated using Kappa statistics.

For analysis of optimal reconstruction intervals, patients were subdivided into four groups according to mean heart rates (Group A, heart rate $<60$ beats per minute; Group B, heart rate $<60-70$ beats per minute; Group C, heart rate $70-80$ beats per minute; Group D, heart rate $\geq 80$ beats per minute). Linear regression with $95 \%$ individual prediction intervals and Pearson correlation analysis were used to assess the relationship between heart rate and image quality. The radiation dose at different heart rates was tested by Student-Newman-Keuls. Reduction of radiation dose by applying the predicted triggering windows was evaluated by $t$-test. The result of invasive coronary angiography was used as the reference standard to calculate the sensitivity, specificity, positive predictive value, and negative predictive value of CT angiography.

\section{Results}

\section{Study patients}

The average heart rate of 76 patients during scanning was $71.2 \pm 13.2$ (range 51-128) beats per minute; 12 patients in Group A, $54.2 \pm 2.8$ (range 51-59) beats per minute; 24 patients in Group B, $64.8 \pm 2.8$ (range 60-69) beats per minute; 26 patients in Group C, $74.2 \pm 2.7$ (range 70-79) beats per minute; 14 patients in Group D, $91.3 \pm 13.3$ (range 80-128) beats per minute. 


\section{Optimal windows for image reconstruction}

Interobserver agreement for determining the image quality of different intervals was moderate (Kappa $=0.70$ ).

For subgroup analysis, a diastolic trough was found around $70 \%$ in Group A, with the systolic trough located at different intervals for the three vessels (right coronary artery $30 \%$, left anterior descending artery $20 \%$, left circumflex artery $20 \%$ ). The quality reconstructed at diastole were better than that at systole (right coronary artery, $P<0.001$; left anterior descending artery, $P=0.001$; left circumflex artery, $P<0.001$ ). In Group B, the trough in diastole was still located around $70 \%$ in all three vessels, while the location of the systolic trough shifted to later phases (right
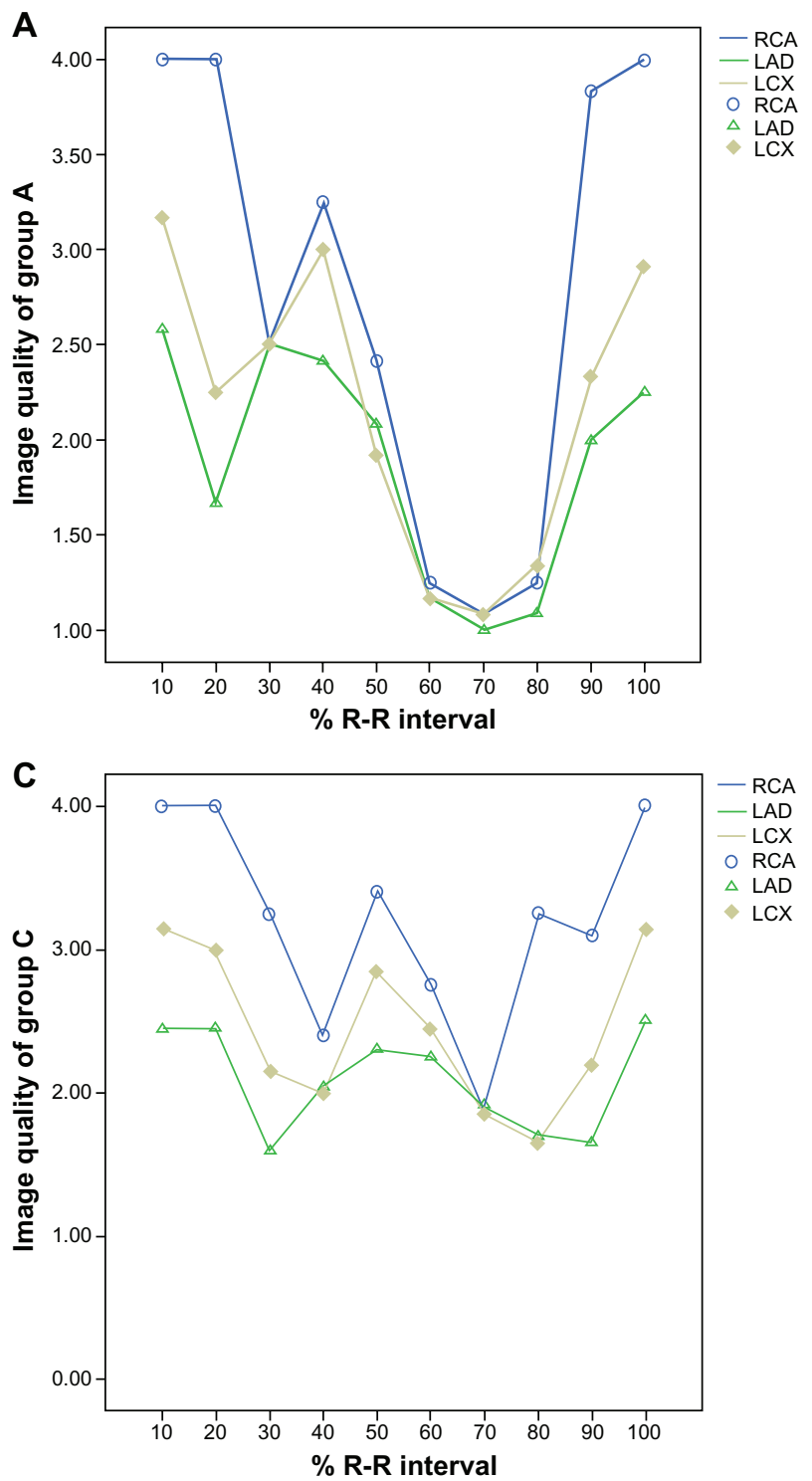

coronary artery $40 \%$, left anterior descending artery $30 \%$, left circumflex artery $30 \%$ ). The quality reconstructed at diastole was better than that at systole (right coronary artery, $P<0.001$; left anterior descending artery, $P=0.03$; left circumflex artery, $P<0.001)$. In Group $\mathrm{C}$, the systolic trough still located at $30 \%-40 \%$, while the diastolic trough located at $70 \%-90 \%$. The quality of the right coronary artery and left circumflex artery reconstructed at diastole was better than that at systole (right coronary artery, $P=0.02$; left circumflex artery, $P=0.04)$. However, there was no significant difference for the left anterior descending artery $(P=0.78)$. In Group D, the systolic trough located around $40 \%$, while the diastolic trough of the right coronary artery appeared at $80 \%$, left anterior descending artery at $90 \%$, and left circumflex
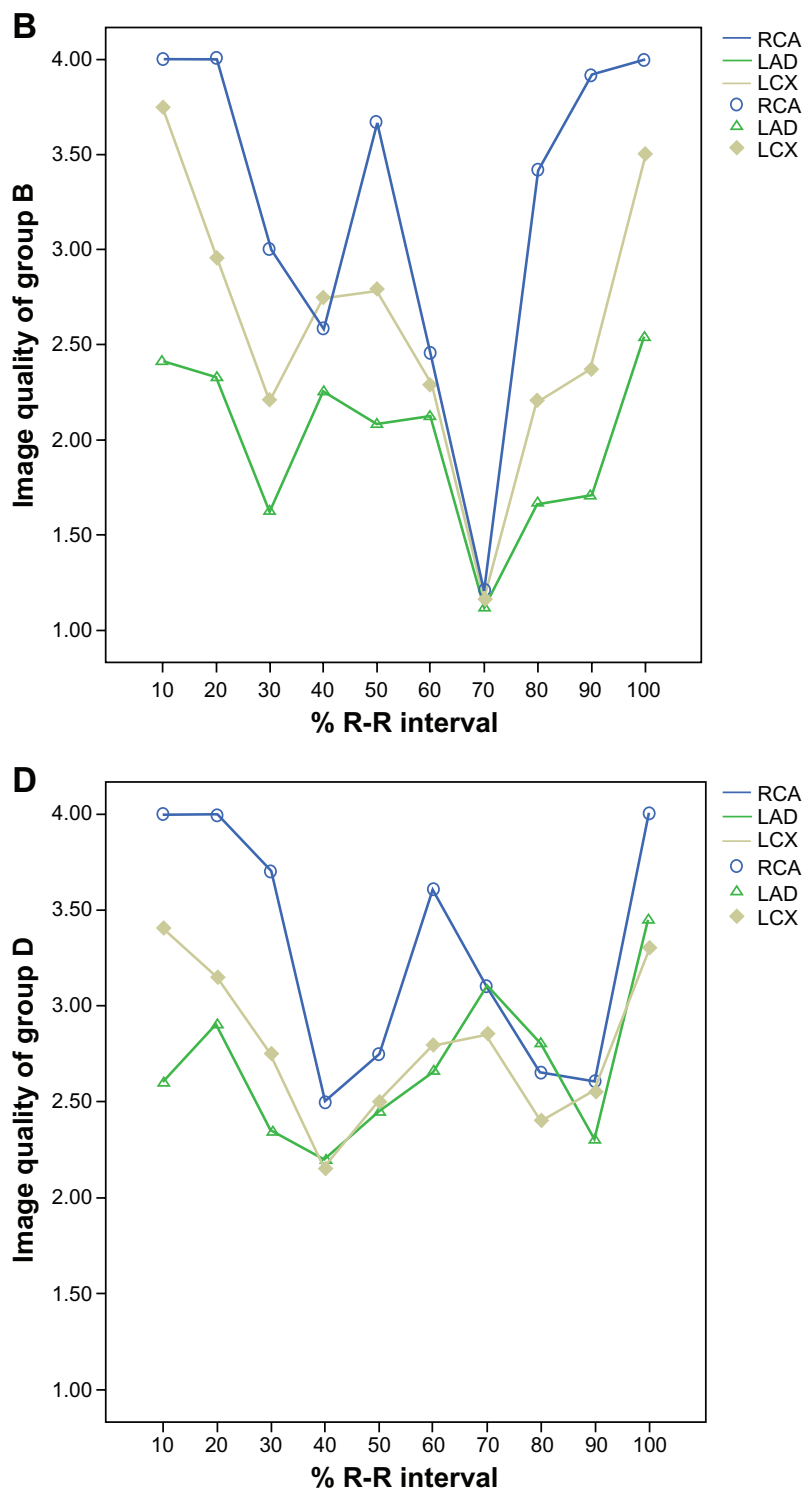

Figure 2 Graphs show image quality of three coronary arteries against time in percentages of R-R interval. Patients were grouped according to heart rate (Group A, heart rate $<60$ beats per minute; Group B, heart rate 60-70 beats per minute; Group C, heart rate 70-80 beats per minute; Group D, heart rate $\geq 80$ beats per minute). Abbreviations: RCA, right coronary artery; LAD, left anterior descending; LCX, left circumflex. 
artery at $80 \%$. There was no significant difference between systole and diastole for the three vessels (right coronary artery, $P=0.37$; left anterior descending artery, $P=0.59$; left circumflex artery, $P=0.17$, Figure 2 ).

After image reconstruction by $1 \%$ intervals around the $10 \%$ intervals with fewest motion artifacts to determine the optimal image quality in both systole and diastole, we found that, as heart rate increased, both the score of optimal image quality in systole and diastole continuously increased ( $\mathrm{r}=0.74, P<0.001 ; \mathrm{r}=0.27, P=0.02$ ). However, the image quality at diastole deteriorated faster than that at systole. According to the linear equation, the cutoff heart rate at which the optimal reconstruction interval turned from diastole to systole was 89.6 beats per minute (Figure 3).

On the basis of analysis of the regulation of image quality score in the different heart rate groups, the optimal exposure phases were determined as follows: when heart rate was $<70$ beats per minute, the exposure windows should be preset at $60 \%-80 \%$; if $70-90$ beats per minute, the preset intervals should be $70 \%-90 \%$; if $\geq 90$ beats per minute, the optimal exposure windows should be $30 \%-50 \%$.

\section{Influence of optimal exposure windows on reduction of radiation dose, image quality, and diagnostic accuracy}

The radiation dose for patients scanned with prospective ECG gating was significantly lower than that of retrospective ECG gating (5.9 versus $12.9 \mathrm{mSv}, P<0.001$ ). The data for subanalysis were shown in Table 2 . The dose was significantly reduced by applying the prospective triggering windows,

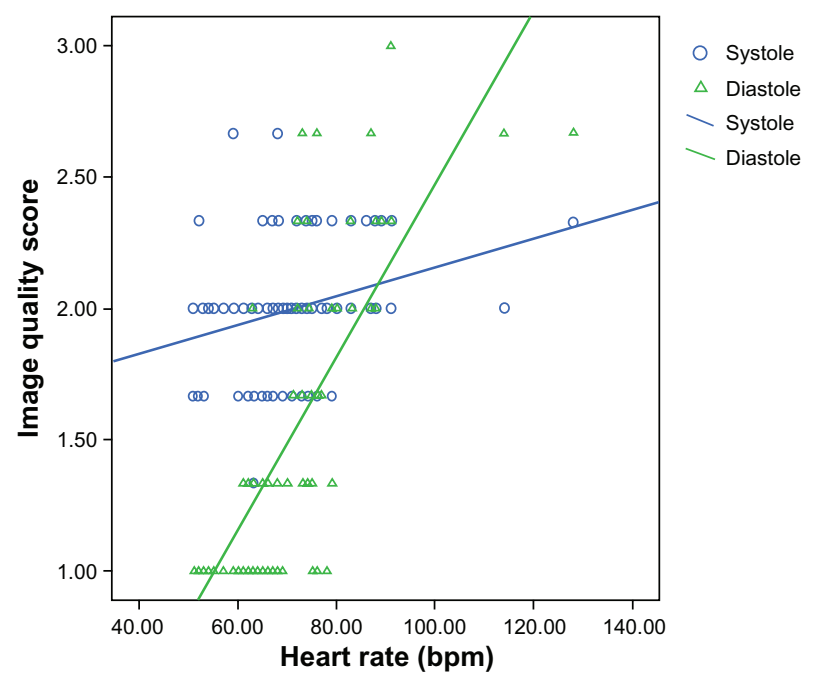

Figure 3 Graph shows that the score of optimal image quality in both systole and diastole continuously increased with higher heart rates.

Notes: $\circ=$ data for systolic reconstructions, $\Delta=$ data for diastolic reconstructions. while the image quality did not deteriorate. However, because two or three heart beats were needed when heart rate was higher than 70 beats per minute, the radiation dose increased with increasing heart rate for both retrospective and prospective ECG gating $(\mathrm{r}=0.64, P<0.001$ for retrospective ECG gating; $\mathrm{r}=0.59, P<0.001$ for prospective ECG gating).

Significant coronary artery stenosis was presented in 49 vessel segments of the 47 patients who underwent invasive coronary angiography. On the basis of a per segment analysis, overall sensitivity was $98.0 \%$ (49/50), specificity was $99.2 \%$ (602/607), positive predictive value was $90.7 \%(49 / 54)$, and negative predictive value was $99.8 \%$ (602/603).

\section{Discussion}

Many studies have demonstrated the effect of a narrow ECG pulsing window on prospective ECG gating to reduce exposure dose. ${ }^{6-12}$ However, because of the constant beating of the heart, it is a challenge to find a short tranquil phase for prospective ECG gated imaging of the coronary arteries. The application of prospective ECG gating has been mainly based on patients with a low heart rate. ${ }^{10,11}$ Dewey et $\mathrm{al}^{17}$ expanded the exposure windows to $30 \%-100 \%$ when heart rate was $>65$ beats per minute, which lead to a dose of $12.3 \mathrm{mSv}$. Our results showed that using DVCT, with narrower optimal intervals (heart rate $<70$ beats per minute, $60 \%-80 \%$; 70-90 beats per minute, 70\%-90\%; $\geq 90$ beats per minute. $30 \%-50 \%$ ), the image was still satisfactory for diagnosis, and the patient radiation dose was decreased significantly. When the heart rate was $>70$ beats per minute, the dose could be reduced to $7.7 \mathrm{mSv}$.

Our results revealed two durations sufficient for image reconstruction, corresponding to the reduced ventricular ejection phase and the slow ventricular filling phase. Overall, the image quality of the systolic reconstruction windows is inferior to that of the diastolic windows. However, image quality in systolic reconstructions did not deteriorate as much as in diastolic reconstructions with increasing heart rate. When the diastolic phase during high heart rate was not sufficient for image reconstruction, the optimal intervals changed to systole. This finding is in agreement with the results of the Husmann and colleagues study about velocity of coronary arteries. ${ }^{28}$

Using 4-MDCT with a gantry rotation speed of $500 \mathrm{msec}$, Herzog et al hypothesized that at heart rates higher than 70 beats per minute, only reconstruction in systole would lead to adequate image quality. ${ }^{21}$ Using a 16-MDCT with a gantry rotation speed of $420 \mathrm{msec}$, Hoffmann et al demonstrated a shift from diastole to systole for optimal image reconstruction between 72 and 80 beats per minute. ${ }^{22}$ Using 64-MDCT 
Table 2 Comparison between retrospective and prospective ECG gating

\begin{tabular}{|c|c|c|c|c|c|c|}
\hline \multirow{2}{*}{$\begin{array}{l}\text { Heart rate } \\
(\mathrm{bpm})\end{array}$} & \multicolumn{2}{|l|}{ Dose (mSv) } & \multirow[t]{2}{*}{$P$} & \multicolumn{2}{|c|}{ Image quality score } & \multirow[t]{2}{*}{$P$} \\
\hline & Retrospective & Prospective & & Retrospective & Prospective & \\
\hline$<70$ & $7.5 \pm 1.5$ & $3.2 \pm 0.8$ & $<0.001$ & $\mathrm{I} . \mathrm{I} \pm 0.2$ & $\mathrm{I} . \mathrm{I} \pm 0.2$ & 0.53 \\
\hline $70-80$ & $14.5 \pm 4.6$ & $5.8 \pm 3.0$ & $<0.001$ & $1.5 \pm 0.3$ & $1.7 \pm 0.5$ & 0.66 \\
\hline $80-90$ & $20.8 \pm 7.5$ & $8.4 \pm 5.3$ & $<0.001$ & $2.2 \pm 0.3$ & $1.9 \pm 0.5$ & 0.14 \\
\hline$>90$ & $20.5 \pm 6.0$ & $10.2 \pm 0.7$ & 0.004 & $2.1 \pm 0.2$ & $2.1 \pm 0.6$ & 0.94 \\
\hline
\end{tabular}

Abbreviations: bpm, beats per minute; ECG, electrocardiographic.

and a gantry rotation speed of $370 \mathrm{msec}$, Leschka et al observed that the shift occurred at a heart rate of 85.5 beats per minute. ${ }^{23}$ Although the gantry rotation speed of DVCT is similar with that of 64-MDCT, the cutoff heart rate improves to 89.6 beats per minute. The increase in heart rate threshold could be explained by the fact that because vessel flow is maximized in diastole, so theoretically the angiogram of the coronary arteries should be performed during diastole. ${ }^{29}$ For DVCT, when heart rate was $>80$ beats per minute, a three heart beat acquisition scan mode was used. With a gantry rotation speed of $350 \mathrm{msec}$, the temporal resolution can reach $58.3 \mathrm{msec}$.

However, because multiple heart beats are needed during scanning, the exposure dose increased significantly with higher heart rates, even using prospective ECG gating.

Our study had some limitations. First, we did not analyze diagnostic accuracy. However, that value can be calculated only if corresponding coronary angiography is available, which was not the case for many of our patients. Second, the results of our study may not be applicable to patients with severe heart rate variability because they are better reconstructed using the absolute timing approach. However, the analysis of our study was based on a relative timing method. Third, image quality scoring might have been influenced by subjectivity bias. Fourth, our results apply only to DVCT and may be different from those obtained using other scanners.

In conclusion, DVCT has the potential to provide high image quality at a wide range of heart rates using an optimized ECG pulsing window. However, it is recommended to control the heart rate below 70 beats per minute, if possible, to decrease the radiation dose.

\section{Disclosure}

The authors report no conflicts of interest in this work.

\section{References}

1. Einstein A, Henzlova M, Rajagopalan S. Estimating risk of cancer associated with radiation exposure from 64-slice computed tomography coronary angiography. JAMA. 2007;298:317-323.
2. Coles DR, Smail MA, Negus IS, et al. Comparison of radiation doses from multislice computed tomography coronary angiography and conventional diagnostic angiography. J Am Coll Cardiol. 2006;47: $1840-1845$.

3. Redberg R, Walsh J. Pay now, benefits may follow - the case of cardiac computed tomographic angiography. N Engl J Med. 2008;359: 2309-2311.

4. Hausleiter J, Meyer T, Hermann F, et al. International prospective multicenter study on radiation dose estimates of coronary $\mathrm{CT}$ angiography in daily practice; ${ }^{a}$ The PROTECTION I study. J Am Coll Cardiol. 2008;2: S19-S20.

5. Einstein AJ, Henzlova MJ, Rajagopalan S. Estimating risk of cancer associated with radiation exposure from 64-slice computed tomography coronary angiography. JAMA. 2007;298:317-323.

6. Paul JF, Abada HT. Strategies for reduction of radiation dose in cardiac multislice CT. Eur Radiol. 2007;17:2028-2037.

7. Husmann L, Valenta I, Gaemperli O, et al. Feasibility of low-dose coronary CT angiography: First experience with prospective ECGgating. Eur Heart J. 2008;29:191-197.

8. Horiguchi J, Kiguchi M, Fujioka C, et al. Radiation dose, image quality, stenosis measurement, and CT densitometry using ECGtriggered coronary 64-MDCT angiography: A phantom study. AJR Am J Roentgenol. 2008;190:315.

9. Earls J, Berman E, Urban B, et al. Prospectively gated transverse coronary CT angiography versus retrospectively gated helical technique: Improved image quality and reduced radiation dose. Radiology. 2008; 246:742-753.

10. Hirai N, Horiguchi J, Fujioka C, et al. Prospective versus retrospective ECG-gated 64-detector coronary CT angiography: Assessment of image quality, stenosis, and radiation dose. Radiology. 2008;248:424-430.

11. Shuman W, Branch K, May J, et al. Prospective versus retrospective ECG gating for 64-detector $\mathrm{CT}$ of the coronary arteries: Comparison of image quality and patient radiation dose. Radiology. 2008;248:431-437.

12. Weustink A, Neefjes L, Kyrzopoulos S, et al. Impact of heart rate frequency and variability on radiation exposure, image quality, and diagnostic performance in dual-source spiral CT coronary angiography. Radiology. 2009;253:672-680.

13. Rybicki FJ, Otero HJ, Steigner ML, et al. Initial evaluation of coronary images from 320-detector row computed tomography. Int J Cardiovasc Imaging. 2008;24:535-546.

14. Steigner ML, Otero HJ, Cai T, et al. Narrowing the phase window width in prospectively ECG-gated single heart beat 320-detector row coronary CT angiography. Int $J$ Cardiovasc Imaging. 2009;25:85-90.

15. Hoe J, Toh KH. First experience with 320-row multidetector CT coronary angiography scanning with prospective electrocardiogram gating to reduce radiation dose. J Cardiovasc Comput Tomogr. 2009;3: 257-261.

16. Hein PA, Romano VC, Lembcke A, May J, Rogalla P. Initial experience with a chest pain protocol using 320-slice volume MDCT. Eur Radiol. 2009;19:1148-1155.

17. Dewey M, Zimmermann E, Deissenrieder F, et al. Noninvasive coronary angiography by 320 -row computed tomography with lower radiation exposure and maintained diagnostic accuracy: Comparison of results with cardiac catheterization in a head-to-head pilot investigation. Circulation. 2009;120:867-875. 
18. Kitagawa K, Lardo A, Lima J, George R. Prospective ECG-gated 320 row detector computed tomography: Implications for CT angiography and perfusion imaging. Int J Card Imaging. 2009;25:201-208.

19. Dewey M, Teige F, Schnapauff D, et al. Noninvasive detection of coronary artery stenoses with multislice computed tomography or magnetic resonance imaging. Ann Intern Med. 2006;145:407-415.

20. Leber AW, Knez A, Becker A, et al. Accuracy of multidetector spiral computed tomography in identifying and differentiating the composition of coronary atherosclerotic plaques: A comparative study with intracoronary ultrasound. J Am Coll Cardiol. 2004;43:1241-1247.

21. Herzog C, Abolmaali N, Balzer JO, et al. Heart-rate-adapted image reconstruction in multidetector-row cardiac CT: Influence of physiological and technical prerequisite on image quality. Eur Radiol. 2002;12: 2670-2678.

22. Hoffmann MH, Shi H, Manzke R, et al. Noninvasive coronary angiography with 16-detector row CT: Effect of heart rate. Radiology. 2005;234: 86-97.

23. Leschka S, Wildermuth S, Boehm T, et al. Noninvasive coronary angiography with 64-section CT: Effect of average heart rate and heart rate variability on image quality. Radiology. 2006;241:378-385.
24. Seifarth H, Wienbeck S, Pusken M, et al. Optimal systolic and diastolic reconstruction windows for coronary CT angiography using dual-source CT. AJR Am J Roentgenol. 2007;189:1317-1323.

25. Brodoefel H, Burgstahler C, Tsiflikas I, et al. Dual-source CT: Effect of heart rate, heart rate variability, and calcification on image quality and diagnostic accuracy. Radiology. 2008;247:346-355.

26. Einstein AJ, Elliston CD, Arai AE, et al. Radiation dose from singleheartbeat coronary CT angiography performed with a 320-detector row volume scanner. Radiology. 2010;254:698-706.

27. Shah A, Gnoj J, Fisher V. Complications of selective coronary arteriography by the Judkins technique and their prevention. Am Heart J. 1975;90:353-359.

28. Husmann L, Leschka S, Desbiolles L, et al. Coronary artery motion and cardiac phases: Dependency on heart rate - implications for CT image reconstruction. Radiology. 2007;245:567-576.

29. Hozumi T, Yoshida K, Akasaka T, et al. Noninvasive assessment of coronary flow velocity and coronary flow velocity reserve in the left anterior descending coronary artery by Doppler echocardiography: Comparison with invasive technique. J Am Coll Cardiol. 1998;32: 1251-1259.
Reports in Medical Imaging

\section{Publish your work in this journal}

Reports in Medical Imaging is an international, peer-reviewed, open access journal publishing original research, reports, reviews and commentaries on all areas of medical imaging. The manuscript management system is completely online and includes a very quick and fair peer-review system, which is all easy to use.

\section{Dovepress}

Visit http://www.dovepress.com/testimonials.php to read real quotes from published authors. 